\title{
3-吗啉基氟化嗍络合二吡咯甲川类化合物的合成及反应机理研究
}

\author{
贺琳彦胡全子席海涛*孙小强 \\ (常州大学石油化工学院常州 213164)
}

\begin{abstract}
摘要 以对着基苯甲醛和吡咯为原料, 合成了 3-吗啉-8-[4'-(3-吗啉丙氧基)苯基]氟化嗍络合二吡咯甲川和 3-吗啉-8-(4'甲氧基苯基)氟化硼络合二吡咯甲川，其结构经过 ${ }^{1} \mathrm{H}$ NMR 和 LC-MS 分析表征，通过相似性试验和晶体的培养对吗啉直 接取代氟化硼络合二吡咯甲川(BODIPY)类化合物 3-H 的反应机理进行研究.
\end{abstract}

关键词 BODIPY; 合成; 反应机理

\section{Synthesis and Mechanism Study of 3-Morpholin Boron- dipyrrolemethene Derivatives}

\author{
He, Linyan $\quad \mathrm{Hu}$, Quanzi $\quad \mathrm{Xi}$, Haitao* Sun, Xiaoqiang \\ (School of Petrochemical Engineering, Changzhou University, Changzhou 213164)
}

\begin{abstract}
Two new 3-morpholin boron-dipyrrolemethene (BODIPY) derivatives, 4,4-difluoro-8-[4'-(3-morpholinopropoxy)phenyl]-3-morpholin-4-bora-3a,4a-diaza-s-indacene and 4,4-difluoro-8-(4-methoxyphenyl)-3-morpholin-4-bora-3a,4a-diaza$s$-indacene, were designed and synthesized from $p$-hydroxybenzaldehyde and pyrrole. The key intermediates and the target compounds were characterized by ${ }^{1} \mathrm{H}$ NMR and LC-MS. The mechanism of direct functionalization of BODIPY dyes at the 3-position was investigated with similarity experiments and the crystal structure.
\end{abstract}

Keywords BODIPY; synthesis; reaction mechanism

氟化硼络合二吡咯甲川(Boron-dipyrrolemethene, 简称 BODIPY)类荧光染料具有较高的摩尔消光系数、光 学稳定性好、吸收发射波长在可见光区等优点 ${ }^{[1]}$, 因此 它在生物化学 ${ }^{[2 \sim 4]}$ 、新型材料 ${ }^{[5,6]}$ 等方面很高的应用价值. 吗啉具有仲胺基团的典型反应特性, 最重要的反应是仲 胺 $\mathrm{N}$ 原子与醇类、卤化物以及醛和甲酸的联合体等 ${ }^{[7]}$ 的取代反应。

对 8-苯系 BODIPY 类化合物(图 1)的 BODIPY 母体 进行不同的修饰, 可以使此类化合物具有不同的光学性 能. 目前, 主要采用的方法是在 BODIPY 母体的 $\alpha-\mathrm{C}^{[8,9]}$ 或 $\beta$ - $\mathrm{C}^{[10,11]}$ 上引入卤素后再引入修饰基团来修饰 BODIPY 类化合物, 而关于亲核试剂直接取代 BODIPY 母体 3-H 的研究查阅到三篇文献. 由 Wim Dehaen 团队 在强碱的反应环境下, 采用具有易离去基团的亲核试 剂, 运用亲核氢取代反应(VNS)机理, 亲核试剂直接取 代 3-H 生成 BODIPY 衍生物 ${ }^{[12]}$. 此后 Wim Dehaen 团队
还运用醋酸钯催化进行氧化亲核取代反应(ONSN), 在 BODIPY 母体的 3-C 或 3,5-C 上直接引入修饰基团 ${ }^{[13,14]}$. 与 Wim Dehaen 团队所用的 18-冠-6 和乙酸钯等昂贵的 催化剂相比, 本研究运用的氧化亲核取代反应具有反应 条件温和、操作简便、价格低廉的优点.

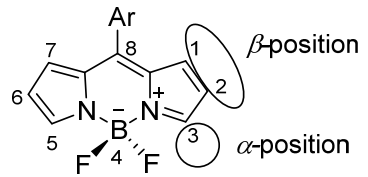

图 18 -苯系 BODIPY 衍生物的化学结构式

Figure 1 Chemical structure of 8-Ar-BODIPY derivatives

\section{1 结果与讨论}

\section{1 反应机理的讨论}

常见的亲核取代反应过程中，离去基团一般是带负 电荷或中性的分子, 氢一般不作为离去基团, 需要额外

\footnotetext{
*E-mail: xht@cczu.edu.cn

Received July 11, 2014; revised September 7, 2014; published online September 18, 2014.

Project supported by the National Natural Science Foundation of China (No.20872051).

国家自然科学基金(No. 20872051)资助项目.
} 
的试剂使之离去. 因此, 我们认为化合物 3 是通过氧化 亲核取代反应合成的. 以吗啉作为亲核试剂, 空气中的 氧气作为氧化剂, 直接取代 BODIPY 母体上 3-H 合成了 具有 3-吗啉基的新型 BODIPY 化合物(Scheme 1). 吗啉 直接取代 BODIPY 母体结构上 3-H 的反应机理如图 2 所示.

由于母体中亚胺键和硼-氟键的存在, 化合物 2 的 BODIPY 母体的 $\alpha$-C 比较活泼. 在碱性条件下, 吗啉上 的氮进攻共轭体系上的 3-C 形成络合物, 使共轭双键移 位, 电子传递给 BODIPY 中的氮正离子, 络合物因负电
荷参与共振，与嗍氟化合物形成稳定的极限结构，再经 氧化脱氢, 生成产物. 由于吗啉单取代的 BODIPY 化合 物具有很强的针化作用 ${ }^{[12]}$, 故没有生成二取代产物. 另 外，以 4-甲氧基苯甲醛和吡咯作为原料、吗啉为亲核试 剂进行相似性实验, 合成相似的化合物 6 (Scheme 2), 经过氢谱和质谱表征化合物 6 同样是 3-H单取代化合物.

化合物 2 的分子式为 $\mathrm{C}_{18} \mathrm{H}_{16} \mathrm{BClF}_{2} \mathrm{~N}_{2} \mathrm{O}$, 相对分子质 量为 360.58. 单晶 $\mathrm{X}$ 射线衍射测试表明，该晶体属于单 斜晶系, 空间群 $P 2_{1} / c$, 晶胞参数分别为 $a=12.9169(14)$

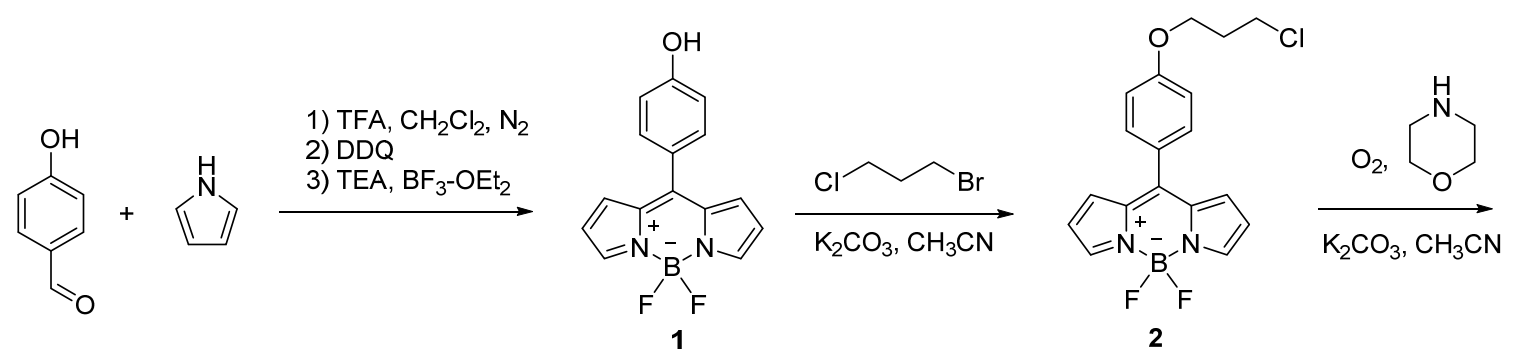<smiles>F[PH](F)(F)n1c(C2=C(c3ccc(OCCCCl)cc3)C=CC2)ccc1N1CCOCC1</smiles>

$3(77 \%)$<smiles></smiles>

$4(20 \%)$<smiles>F[B-]1(F)n2cccc2C(c2ccc(OCCCN3CCOCC3)cc2)=C2C=CC=[N+]21</smiles>

图式 2 化合物 $\mathbf{3}$ 的合成

Scheme 1 Synthetic route of compound 3
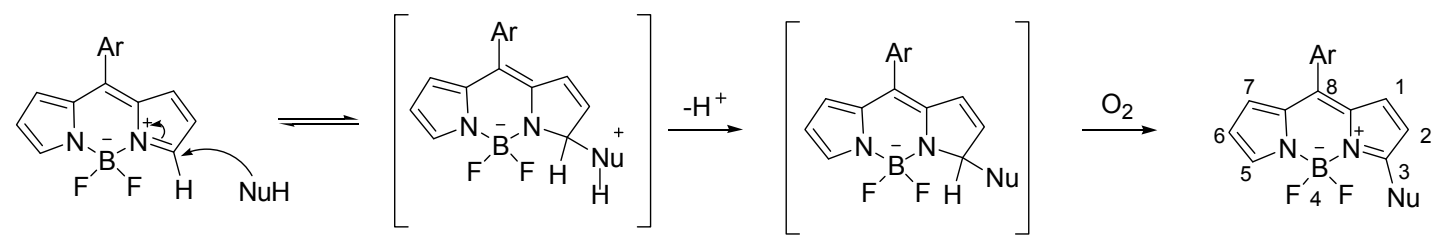

图 2 BODIPY 化合物的氧化亲核取代反应机理

Figure 2 Direct oxidative substitution on BODIPYs
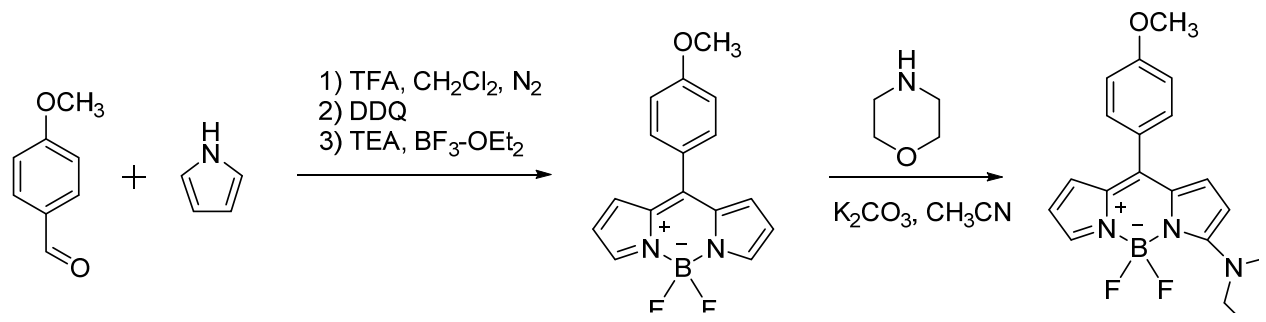

图式 2 化合物 6 的合成

Scheme 2 Synthetic route of compound 6 
$\AA, b=9.0669(10) \AA, c=16.6006(13) \AA, \alpha=90^{\circ}, \beta=$ $119.507(6)^{\circ}, \gamma=90^{\circ}, V=1692.0(3) \AA^{3}, Z=4, D_{\mathrm{m}}=1.416$ $\mathrm{mg} / \mathrm{m}^{3}, D_{\mathrm{c}}=1.416 \mathrm{mg} / \mathrm{m}^{3}, \lambda=0.71073 \AA, \mu=0.254$ $\mathrm{mm}^{-1}, F(000)=744$, BODIPY 母体与苯环之间的二面角 为 $51.76^{\circ}$.

观察化合物 $\mathbf{2}$ 的晶胞堆积图(图 3)发现, 分子间的相 互作用力包括非经典型 $\mathrm{C}-\mathrm{H} \cdots \mathrm{F}$ 和 $\mathrm{C}-\mathrm{H} \cdots \mathrm{Cl}$ 氢 键 ${ }^{[15,16]}$. 首先, $\mathrm{C}-\mathrm{H} \cdots \mathrm{F}$ 氢键使两个平行的分子首尾相 连成环, 包含两分子的环存在两种排列方式, 这两种排 列方式的 BODIPY 母体之间的二面角为 $38.61^{\circ}$. 然后, $\mathrm{C}-\mathrm{H} \cdots \mathrm{Cl}$ 键将这两种环交错连接延伸成锯齿形的一维 链, 晶体由这些一维链堆积而成. 从晶体堆积图看出, 锯齿形的链状结构让 BODIPY 母体上的一个五元环裸 露在边缘, 另一个五元环夹在其他分子的中间, 使得亲 核试剂进攻两个 $\alpha-\mathrm{C}$ 时由于位阻的原因产生了难易之 分, 故亲核试剂更易进攻 3-C 生成单取代产物.

\section{2 结论}

一般 BODIPY 类衍生物 $\alpha$ 位的结构修饰反应条件 都比较复杂, 本研究发现在碳酸钾和乙腈的反应体系 中, 氧气作为氧化剂, 亲核试剂吗啉直接取代 BODIPY 母体上的 3-H 达到修饰结构的效果. 经过对反应机理、 相似性实验以及晶体结构的研究, 证明氧化亲核取代反 应生成了单取代的 3-吗啉基 BODIPY 类化合物，为亲核 试剂直接取代 BODIPY 类衍生物上 $3-\mathrm{H}$ 的反应提供参考 价值.

\section{3 实验部分}

\section{1 仪器与试剂}

XT4-100X 显微熔点测定仪(上海申光仪器仪表有 限公司); AVANCE $500 \mathrm{MHz}$ 型核磁共振仪(Bruker 公司, TMS 为内标); Bruker Autoflex II MALDI-TOF 质谱仪和 Bruker Smart CCD 衍射仪(Bruker Daltonics 公司).

化合物 $\mathbf{1}$ 和化合物 $\mathbf{5}$ 按照文献[17]报道的方法合成, 柱层析用硅胶 $\mathrm{H}$ (青岛海洋化工有限公司), 其余所用试 剂均为国产分析纯.

\section{2 化合物的合成}

\section{2 .1 化合物 $\mathbf{2}$ 的合成}

在反应瓶中依次加入 $200.0 \mathrm{mg}(0.70 \mathrm{~mol})$ 化合物 $\mathbf{1}$, 无水乙腈 $10 \mathrm{~mL}, 1$-溴-3-氯丙烷 $165.3 \mathrm{mg}$ (1.05 mmol)和 碳酸钾 $193.2 \mathrm{mg}(1.4 \mathrm{mmol}), 50{ }^{\circ} \mathrm{C}$ 下搅拌反应. 薄层色 谱(TLC)跟踪至化合物 1 消耗完, 冷却抽滤, 浓缩滤液. 用硅胶柱层析分离提纯[洗脱剂: $V$ (二氯甲烷) $: V$ (石油 醚 $)=1: 10$ ], 得橘黄色固体 $228.5 \mathrm{mg}$, 产率 $90 \%$. m.p. $142.4 \sim 142.5{ }^{\circ} \mathrm{C} ;{ }^{1} \mathrm{H}$ NMR $\left(500 \mathrm{MHz}, \mathrm{CDCl}_{3}\right) \delta: 7.92(\mathrm{~s}$, $2 \mathrm{H}), 7.55(\mathrm{~d}, J=8.7 \mathrm{~Hz}, 2 \mathrm{H}), 7.05(\mathrm{~d}, J=8.7 \mathrm{~Hz}, 2 \mathrm{H}), 6.97$ (d, $J=3.9 \mathrm{~Hz}, 2 \mathrm{H}), 6.55(\mathrm{~d}, J=2.7 \mathrm{~Hz}, 2 \mathrm{H}), 4.23$ (t, $J=5.8$ $\mathrm{Hz}, 2 \mathrm{H}), 3.79(\mathrm{t}, J=6.2 \mathrm{~Hz}, 2 \mathrm{H}), 2.63 \sim 2.10(\mathrm{~m}, 2 \mathrm{H})$.

3.2.2 化合物 3 和 4 的合成

在 $25 \mathrm{~mL}$ 的单口烧瓶中依次加入 $74.4 \mathrm{mg}(0.2$ $\mathrm{mmol}$ )化合物 2, 无水乙腈 $5 \mathrm{~mL}$, 吗啡啉 $1.0 \mathrm{~mL}$ (过量), 碳酸钾 $34.2 \mathrm{mg}$ (2.4 mmol), 室温下敞口体系反应. 薄层

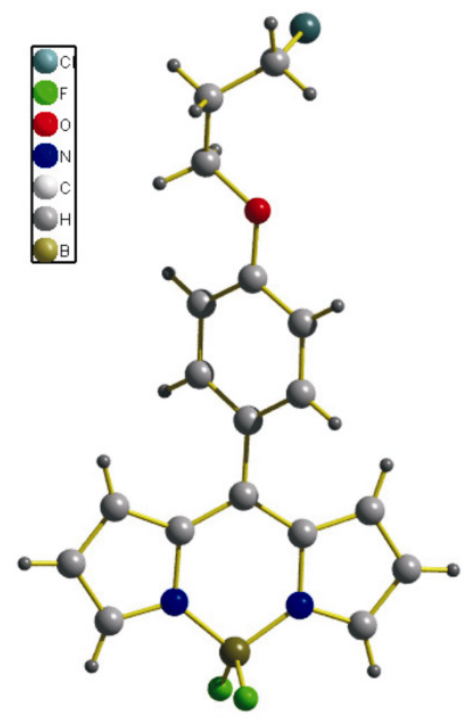

(a)

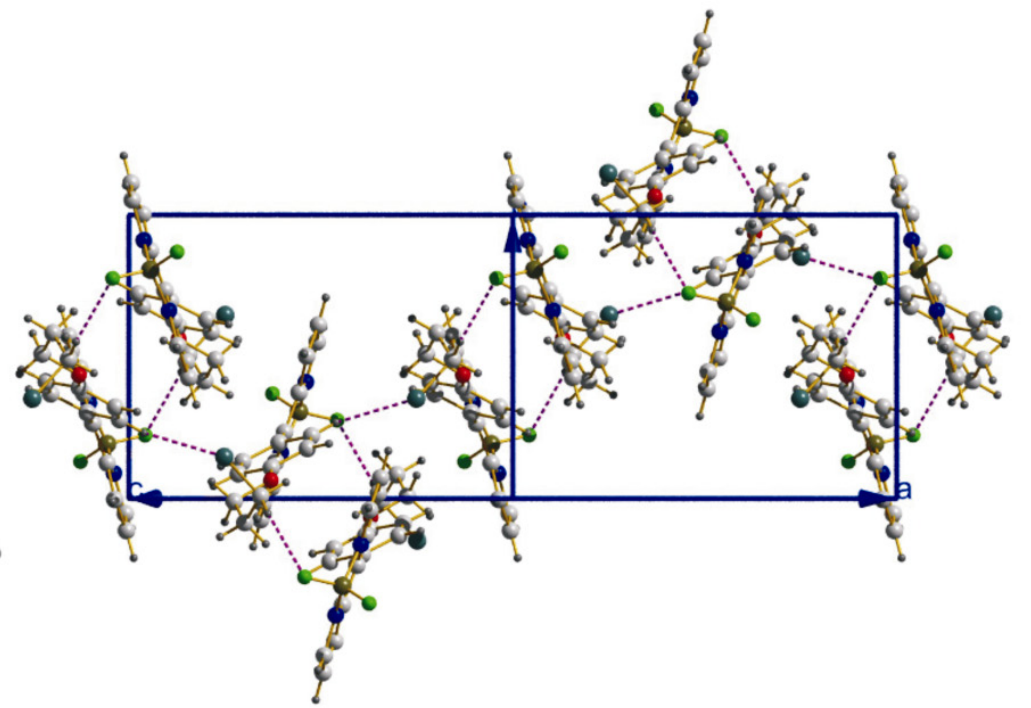

(b)

图 3 化合物 $\mathbf{2}$ 的 X 衍射图

Figure 3 X-ray crystal structure of 2

(a) The molecular structure of 2; (b) A view, in projection down the $b$ axis, of the unit-cell contents of 2 
色谱(TLC)跟踪反应至化合物 $\mathbf{2}$ 消耗完, 冷却抽滤, 浓缩 滤液. 用硅胶柱层析分离提纯 [洗脱剂: $V$ (丙酮) : $V$ (石 油醚 $)=1: 10]$, 收集两组主产物, 分别得化合物 $3(70.4$ $\mathrm{mg}$ )和 4 (20.1 mg). 化合物 3: 产率 77\%. m.p. 182.4 $183.3{ }^{\circ} \mathrm{C} ;{ }^{1} \mathrm{H}$ NMR $\left(500 \mathrm{MHz}, \mathrm{CDCl}_{3}\right) \delta: 7.46$ (s, 1H), 7.41 (d, $J=8.4 \mathrm{~Hz}, 2 \mathrm{H}), 6.98$ (d, $J=8.4 \mathrm{~Hz}, 2 \mathrm{H}), 6.93$ (d, $J=$ $5.0 \mathrm{~Hz}, 1 \mathrm{H}), 6.43(\mathrm{~d}, J=3.0 \mathrm{~Hz}, 1 \mathrm{H}), 6.36(\mathrm{~d}, J=2.3 \mathrm{~Hz}$, $1 \mathrm{H}), 6.21(\mathrm{~d}, J=5.0 \mathrm{~Hz}, 1 \mathrm{H}), 4.19$ (t, $J=5.8 \mathrm{~Hz}, 2 \mathrm{H}), 3.95$ $(\mathrm{d}, J=4.1 \mathrm{~Hz}, 4 \mathrm{H}), 3.89$ (d, $J=4.3 \mathrm{~Hz}, 4 \mathrm{H}), 3.79$ (t, $J=6.2$ $\mathrm{Hz}, 2 \mathrm{H}), 2.36 \sim 2.20$ (m, 2H); MS $m / z(\%): 468.10[\mathrm{M}+$ $23]^{+}$.

化合物 4: 产率 20\%. m.p. $185.6 \sim 186.1{ }^{\circ} \mathrm{C} ;{ }^{1} \mathrm{H}$ NMR (500 MHz, $\left.\mathrm{CDCl}_{3}\right) \delta: 7.46(\mathrm{~s}, 1 \mathrm{H}), 7.40$ (d, $J=8.3$ $\mathrm{Hz}, 2 \mathrm{H}), 6.97$ (d, $J=8.3 \mathrm{~Hz}, 2 \mathrm{H}), 6.93$ (d, $J=4.9 \mathrm{~Hz}, 1 \mathrm{H})$, $6.43(\mathrm{~s}, 1 \mathrm{H}), 6.36(\mathrm{~s}, 1 \mathrm{H}), 6.21(\mathrm{~d}, J=4.8 \mathrm{~Hz}, 1 \mathrm{H}), 4.09$ (t, $J=6.1 \mathrm{~Hz}, 2 \mathrm{H}), 3.95$ (s, 4H), 3.89 (s, 4H), 3.74 (s, 4H), $2.56(\mathrm{t}, J=7.2 \mathrm{~Hz}, 2 \mathrm{H}), 2.49$ (s, 4H), $2.11 \sim 1.94(\mathrm{~m}, 2 \mathrm{H})$; MS $m / z(\%): 497.25[\mathrm{M}+1]^{+}$.

\section{2 .3 化合物 6 的合成}

在 $25 \mathrm{~mL}$ 的单口烧瓶中依次加入 $59.6 \mathrm{mg}(0.2$ $\mathrm{mmol}$ )化合物 5, 无水乙腈 $5 \mathrm{~mL}$, 吗啡啉 $1.0 \mathrm{~mL}$ (过量), 碳酸钾 $34.2 \mathrm{mg}$ (2.4 mmol), 室温下敞口体系反应. 薄层 色谱(TLC)跟踪反应至化合物 $\mathbf{5}$ 消耗完, 冷却抽滤, 浓缩 滤液. 用硅胶柱层析分离提纯 [洗脱剂: $V$ (丙酮) $: V$ (石 油醚 $)=1: 10]$, 收集化合物 $6(54.4 \mathrm{mg})$, 产率 $71 \%$. ${ }^{1} \mathrm{H}$ NMR (500 MHz, $\left.\mathrm{CDCl}_{3}\right) \delta: 7.46(\mathrm{~d}, J=1.0 \mathrm{~Hz}, 1 \mathrm{H}), 7.41$ (dd, $J=6.7,2.0 \mathrm{~Hz}, 2 \mathrm{H}), 6.98$ (d, $J=8.7 \mathrm{~Hz}, 2 \mathrm{H}), 6.93$ (d, $J=5.1 \mathrm{~Hz}, 1 \mathrm{H}), 6.44$ (d, $J=3.0 \mathrm{~Hz}, 1 \mathrm{H}), 6.36$ (d, $J=2.3$ $\mathrm{Hz}, 1 \mathrm{H}), 6.22(\mathrm{~d}, J=5.1 \mathrm{~Hz}, 1 \mathrm{H}), 4.01 \sim 3.93(\mathrm{~m}, 4 \mathrm{H})$, $3.89(\mathrm{~d}, J=8.6 \mathrm{~Hz}, 7 \mathrm{H})$; $\mathrm{MS} m / z(\%): 406.10[\mathrm{M}+23]^{+}$.

\section{3 化合物 2 的晶体培养和结构测定}

将化合物 2 溶解在二氯甲烷和正己烷(体积比为 4:1)重配成适当的稀溶液于三角雉形瓶中, 用保鲜膜 封好, 室温静置待溶剂自然挥发的所需晶体取尺寸为 $0.25 \mathrm{~mm} \times 0.20 \mathrm{~mm} \times 0.19 \mathrm{~mm}$ 的单晶, 置于 Bruker Smart CCD 衍射仪上进行衍射实验. 数据收集条件为: 293(2) K, 采用 $\mathrm{Mo} \mathrm{K} \alpha(\lambda=0.71073 \AA)$ 射线和以 $\omega / 2 \theta$ 可 变扫描方式, 收集范围 $1.8^{\circ} \leqslant \theta \leqslant 26.0^{\circ},-13 \leqslant h \leqslant 15$,
$-10 \leqslant k \leqslant 11 ，-18 \leqslant l \leqslant 18$. 总共收集 9977 个衍射点, 其中 3323 个 $\left(R_{\mathrm{int}}=0.047\right)$ 独立衍射点, 其中 $I>2.0 \sigma(I)$ 的 可观察点 2498 个用于晶体结构分析, 对全部非氢原子 的坐标及各向异性参数用 SHELXS-97 程序以最小二乘 法修正 $F^{2}$ 进行精修, 最终偏离因子 $R_{1}=0.0598, w R_{2}=$ 0.1951. 化合物 2 在剑桥晶体结构数据库的编号为 CCDC 1013007.

辅助材料(Supporting Information) 化合物 $\mathbf{1} \sim 6$ 的 ${ }^{1} \mathrm{H}$ NMR 谱图、化合物 3, 4, 6 的 MS 谱图以及化合物 2 晶 体的键长和键角数据. 这些材料可以免费从本刊网站 (http://sioc-journal.cn/)上下载.

\section{References}

[1] Loudet, A.; Burgess. K. Chem. Rev. 2007, 107, 4891.

[2] Lu, Z. T.; Zhang, X. G.; Wu, Z. M.; Zhai, T. T.; Xue, Y. A.; Mei, L.; Li, C. X. RSC Adv. 2014, 4, 19495.

[3] Zhao, T. T.; Chen, Q. Y.; Wang, P. D.; Chen Z P. RSC Adv. 2014 4, 10390.

[4] Ozdemir, T.; Sozmen, F.; Mamur, S.; Tekinay, T.; Akkaya, E. U. Chem. Commun. 2014, 50, 5455.

[5] Mirloup, A.; Retailleau, P.; Ziessel, R. Tetrahedron Lett. 2013, 54, 4456.

[6] Imai, G.; Kogure, H.; Ogiso, A.; Misawa, T.; Nishimoto, T.; Tsukahara, H.; Takuma, K. JP 2000001509, 2000 [Chem. Abstr. 2000, 132, 64968].

[7] Mesropyan, E. G.; Ambartsumyan, G. B.; Avetisyan, A. A.; Galstyan, A. S.; Kirakosyan, A. N. Khimiya Geterotsiklicheskikh Soedinenii, 2005, (11), 1691.

[8] Dilek, Ö.; Bane, S. L. Tetrahedron Lett. 2008, 49, 1413.

[9] Dilek, Ö.; Bane, S. L. Bioorg. Med. Chem. Lett. 2009, 19(284), 6911.

[10] Imai, G.; Kogure, H.; Ogiso, A.; Misawa, T.; Nishimoto, T.; Tsukahara, H.; Takuma, K. JP 2000039716, 2000 [Chem. Abstr. 2000, 132, 144418].

[11] Yogo, T.; Urano, Y.; Ishitsuka, Y.; Maniwa, F.; Nagano, T. J. Am. Chem. Soc. 2005, 127, 12162.

[12] Leen, V.; Auweraer, M. V.; Boens, N.; Dehaen, W. Org. Lett. 2011, 13(6), 1470.

[13] Leen, V.; Gonzalvo, V. Z.; Deborddraeve, W. W.; Boens, N.; Dehaen, W. Chem. Commun. 2010, 46, 4908.

[14] Verbelen, B.; Leen, V.; Wang, L. N.; Boens, N.; Dehaen, W. Chem. Commun. 2012, 48, 9129.

[15] Steiner, T. Angew. Chem., Int. Ed. 2002, 41, 48

[16] Calhorda, M. J. Chem. Commun. 2000, 801. Littler, B. J.; Miller, M. A.; Hung, C. H.; O'Shea, D. F.; Boyle, P. D.; Lindsey, J. S. J. Org. Chem. 1999, 64, 1391. 